TRANSACTIONS OF THE

AMERICAN MATHEMATICAL SOCIETY

Volume 281. Number 2, February 1984

\title{
ON THE PATHS OF SYMMETRIC STABLE PROCESSES
}

BY

\author{
BURGESS DAVIS ${ }^{1}$
}

\begin{abstract}
It is shown that if $X(t), t \geqslant 0$, is a symmetric stable process of index $\alpha$, $0<\alpha<2$, then $\sup _{t} \liminf _{h \downarrow 0}(X(t+h)-X(t)) h^{-1 / \alpha}=\infty$ a.s. This settles a question of Fristedt about strictly stable subordinators.
\end{abstract}

1. Introduction. This paper studies the paths of stable processes, that is, processes with stable, stationary and independent increments. We will always work with the version of a process which has right continuous paths with left limits, and rule out the trivial case that a process is identically zero. Edwin Perkins has recently proved, in [7], that if $Z(t), t \geqslant 0$, is a symmetric stable processes of index $\alpha \in(0,2)$, then there is a constant $c_{1} \in(0, \infty)$ such that

$$
\inf _{t} \limsup _{h \downarrow 0} \frac{|Z(t+h)-Z(t)|}{h^{1 / \alpha}}=c_{1} \quad \text { a.s. }
$$

Here we show

$$
\sup \liminf _{h \downarrow 0} \frac{Z(t+h)-Z(t)}{h^{1 / \alpha}}=\infty \quad \text { a.s. }
$$

This contrasts with the $\alpha=2$ (Brownian motion) case. If $W(t), t \geqslant 0$, is a standard Wiener process then both

$$
\inf _{t} \limsup _{h \downarrow 0} \frac{|W(t+h)-W(t)|}{h^{1 / 2}}=1 \quad \text { a.s., }
$$

and

$$
\sup _{t} \liminf _{h \downarrow 0} \frac{W(t+h)-W(t)}{h^{1 / 2}}=1 \quad \text { a.s. }
$$

Equality (1.3) was proved independently by P. Greenwood and E. Perkins [5] and by the author [1] and is a refinement of results of Dvoretsky [3] and Kahane [6]. Equality (1.4) is proved in [1].

Let $X(t), t \geqslant 0$, be a strictly stable subordinator, that is, a stable process satisfying

$$
E e^{-\lambda X(t)}=e^{-c t \lambda^{\alpha}}
$$

Received by the editors December 30, 1982.

1980 Mathematics Subject Classification. Primary $60 \mathrm{~J} 30$.

Key words and phrases. Symmetric stable process, sample path.

${ }^{1}$ Research supported by National Science Foundation Grant No. MCS 8201128. 
for a positive scale constant $c$, and an index constant $\alpha \in(0,1)$. Strictly stable subordinators have nondecreasing sample paths which increase only by jumps, and every increasing stable process $Y(t), t \geqslant 0$, can be decomposed $Y(t)=H(t)+k t$, where $H(t)$ is a strictly stable subordinator and $k \geqslant 0$. Bert Fristed has shown [4] that, if $X(t)$ is a strictly stable subordinator of index $\alpha$, then there are constants $c_{2}$, $c_{3}, c_{4}$ in $(0, \infty)$ such that

$$
\begin{gathered}
\inf _{t} \limsup _{h \rightarrow 0} \frac{|X(t+h)-X(t)|}{|h|^{1 / \alpha}}=c_{2} \quad \text { a.s., } \\
\inf _{t} \limsup _{h \downarrow 0} \frac{X(t+h)-X(t)}{h^{1 / \alpha}}=c_{3} \quad \text { a.s., }
\end{gathered}
$$

and

$$
\sup _{t} \liminf _{h \rightarrow 0} \frac{|X(t+h)-X(h)|}{|h|^{1 / \alpha}}=c_{4} \text { a.s. }
$$

Fristedt did not decide whether the "natural" (1.8) holds. Equality (1.2) shows it does not. Since the difference of two independent identically distributed strictly stable subordinators is a symmetric stable process, (1.2) implies

$$
\sup _{t} \liminf _{h \downarrow 0} \frac{X(t+h)-X(t)}{h^{1 / \alpha}}=\infty \text { a.s. }
$$

Even though (1.8) follows from (1.2), we prove it separately, and first, in $\S 2$, since this proof has the ideas but not the technicalities of the proof of (1.2). The proofs use techniques from [1].

The proof of (1.8) does not show there is almost surely a time $s$ such that $\liminf _{t \downarrow 0}(X(t+s)-X(s)) t^{-1 / \alpha}=\infty$, nor does the proof of (1.2) give this information about $Z(t)$. We cannot decide whether there are such times and do not even have a guess. We do show, in $\$ 4$, that the Hausdorff dimension of these times is almost surely zero.

2. Strictly stable subordinators. In this section $X(t), t \geqslant 0$, will be a strictly stable subordinator of index $\alpha \in(0,1)$, which is considered fixed. For definiteness we take the scale constant $c$ to be 1 . Define $\mathscr{F}_{t}^{X}=\mathscr{F}_{t}$, for $t>0$, to be the $\sigma$-field $\sigma(X(s), 0<s \leqslant t)$. Frequent use will be made of the scaling property of stable processes, which is that, for any positive constant $k$, the process $k^{-1 / \alpha} X(k t), t \geqslant 0$, has the same distribution as the process $X(t), t \geqslant 0$. We will also need the following welll-known estimate on the tail of $X(1)$. There is a positive constant $\mu$ such that

$$
\liminf _{y \rightarrow \infty} P(X(1)>y) y^{\alpha} \geqslant \mu .
$$

This follows from the fact that the Levy measure of $X(1)$ is a constant $\theta$ times $t^{-(1+\alpha)} I(t>0) d t$, so that the probability that $X(t), 0 \leqslant t \leqslant 1$, makes a jump of magnitude exceeding $x$ is $1-\exp \left(-\int_{x}^{\infty} \theta t^{-(1+\alpha)} d t\right) \sim \mu x^{-\alpha}$, where $\mu=\theta / \alpha$. In this section we prove the following theorem. 
THEOREM 2.1. Given $\lambda>0$ there is an $r<\infty$ such that

$$
P\left(\exists t \in[0,1]: \lambda h^{1 / \alpha} \leqslant X(t+h)-X(t) \leqslant r h^{1 / \alpha} \text { for all } h \in(0,1]\right)>0 .
$$

Theorem 2.1 clearly implies (1.8), since the probability in (2.2) is the same if " $\exists t \in[0,1]$ " is replaced by " $\exists t \in[a, a+1]$ ". It also gives half of Fristedt's result (1.6), the " $<\infty$ " half.

Proof. Given $0<a<b$ and $k$ a positive integer let $E(a, b, k)=\{\exists t \in[0,1]$ : $\left.a h^{1 / \alpha}<X(t+h)-X(t)<b h^{1 / \alpha} \forall h \in\left[k^{-1}, 1\right]\right\}$. If some sample point $\omega$ is in $E(a, b, k)$ for infinitely many $k$, that is, if for a subsequence $k_{i}, i \geqslant 1$, there exist times $t\left(k_{i}\right)$ satisfying

$$
a h^{1 / \alpha}<X\left(t\left(k_{i}\right)+h\right)-X\left(t\left(k_{i}\right)\right)<b h^{1 / \alpha} \text { for all } h \text { in }\left[k_{i}^{-1}, 1\right],
$$

then if $\tau(\omega)=\tau$ is any cluster point of the $t\left(k_{i}\right), i \geqslant 1$, it is not hard to show that

$$
a h^{1 / \alpha} \leqslant X(\tau+h)-X(\tau) \leqslant b h^{1 / \alpha} \text { for all } h \text { in }[0,1] .
$$

Note that both the upper and lower bounds in (2.3) are needed to get the lower bound in (2.4). The upper bound guarantees that the times $t\left(k_{i}\right)$ do not increase to the time of a jump of $X(t)$. Thus, to prove (2.2) it suffices to prove that given $\lambda>0$ there is $r<\infty$ such that

$$
\limsup _{n \rightarrow \infty} P E(\lambda, r, n)>0 .
$$

For $0<a<b$ let $T_{a, b}=\inf \left\{t \geqslant 1: X(t) \notin\left(a t^{1 / \alpha}, b t^{1 / \alpha}\right)\right\}$.

LeMma 2.2. Given $\lambda>0$ there exists $r<\infty$ and $a p \in(0,1)$ such that $E T_{\lambda, r}^{p}=\infty$.

Proof. We consider $\lambda$ as fixed, and put $\theta=2^{-1}\left(2^{\alpha}-1\right)^{1 / \alpha}$. Let $p \in(0,1)$ and $y<\infty$ satisfy

$$
E\left[\lambda^{-\alpha}(\gamma X(1)+\lambda)^{\alpha}-1\right]^{p} I(2 \lambda<\gamma X(1)+\lambda<y)>2,
$$

for all constants $\gamma$ in $[\theta, 1]$. It is not difficult to show using (2.1) that there exist such $p$ and $y$. Note (2.1) immediately gives $E X(1)^{\alpha}=\infty$, so that $E X(1)^{\alpha p} \rightarrow \infty$ as $p \uparrow 1$.

Define stopping times $\tau_{i}, i \geqslant 0$, by $\tau_{0}=1, \tau_{1}=(X(1) / \lambda)^{\alpha}$ if $X(1) \in(2 \lambda, y)$, $\tau_{1}=1$ otherwise, and, for $i \geqslant 2, \tau_{i}=\tau_{i-1}$ if either $\tau_{i-1}=\tau_{i-2}$ or if $X\left(\tau_{i-1}\right) \notin$ $\left(2 \lambda \tau_{i-1}^{1 / \alpha}, y \tau_{i-1}^{1 / \alpha}\right)$, and

$$
\tau_{i}=\left(X\left(\tau_{i-1}\right) / \lambda\right)^{\alpha}, \quad \text { otherwise, }
$$

and put $\tau=\lim _{n \rightarrow \infty} \tau_{n}$ and $N=\inf \left\{n: \tau=\tau_{n}\right\}$. We have $\tau=\tau_{N} \leqslant(y / \lambda)^{\alpha} \tau_{N-1}$, and $\tau_{N-1} \leqslant T_{\lambda, r}$ where $r=y^{2} / \lambda$. Thus, to show $E T_{\lambda, r}^{p}=\infty$ it is sufficient to show $E \tau^{p}=\infty$, which will be accomplished by showing that $E\left(\tau_{i}-\tau_{i-1}\right)^{p} \rightarrow \infty$ as $i \rightarrow \infty$.

Now on $\left\{\tau_{k}>\tau_{k-1}\right\}$,

$$
\begin{aligned}
X\left(\tau_{k}\right) & =X\left(\tau_{k-1}\right)+\left(X\left(\tau_{k}\right)-X\left(\tau_{k-1}\right)\right)=\lambda \tau_{k}^{1 / \alpha}+\left(X\left(\tau_{k}\right)-X\left(\tau_{k-1}\right)\right) \\
& =\tau_{k}^{1 / \alpha}(\lambda+s Z),
\end{aligned}
$$


where $s=\left(\tau_{k}-\tau_{k-1}\right)^{1 / \alpha} / \tau_{k}^{1 / \alpha}$ and $Z=\left(X\left(\tau_{k}\right)-X\left(\tau_{k-1}\right)\right) /\left(\tau_{k}-\tau_{k-1}\right)^{1 / \alpha}$. Here $\theta \leqslant s \leqslant 1$ since $\tau_{k} \geqslant 2^{\alpha} \tau_{k-1}$, and, since $\tau_{k}$ is $\mathscr{F}_{\tau_{k-1}}$ measurable, so is $s$. Furthermore, given $\tau_{k}>\tau_{k-1}, Z$ is independent of $\widetilde{F}_{\tau_{k-1}}$ and has the same distribution that $X(1)$ has. We have

$$
\begin{aligned}
E\left(\tau_{k+1}-\tau_{k}\right)^{p}=E\left(\left(X\left(\tau_{k}\right) / \lambda\right)^{\alpha}-\tau_{k}\right)^{p} I\left(X\left(\tau_{k}\right) \in\left(2 \lambda \tau_{k}^{1 / \alpha}, y \tau_{k}^{1 / \alpha}\right)\right) I\left(\tau_{k}>\tau_{k-1}\right) \\
=E\left(\tau_{k}(\lambda+s Z)^{\alpha} \lambda^{-\alpha}-\tau_{k}\right)^{p} I(\lambda+s Z \in(2 \lambda, y)) I\left(\tau_{k}>\tau_{k-1}\right) \\
\left.=E E\left(\tau_{k}(\lambda+s Z)^{\alpha} \lambda^{-\alpha}-\tau_{k}\right)^{p} I(\lambda+s Z \in(2 \lambda, y)) I\left(\tau_{k}>\tau_{k-1}\right) \mid \mathscr{F}_{\tau_{k-1}}\right) \\
=E \tau_{k}^{p} I\left(\tau_{k}>\tau_{k-1}\right) E\left((\lambda+s Z)^{\alpha} \lambda^{-\alpha}-1\right)^{p} I\left(\lambda+s Z \in(2 \lambda, y) \mid \mathscr{F}_{\tau_{k-1}}\right) \\
\geqslant E \tau_{k}^{p} I\left(\tau_{k}>\tau_{k-1}\right)^{2} \geqslant 2 E\left(\tau_{k}-\tau_{k-1}\right)^{p} .
\end{aligned}
$$

This implies $E\left(\tau_{i}-\tau_{i-1}\right)^{p} \rightarrow \infty$ as $i \rightarrow \infty$.

The next lemma, which we have not been able to find elsewhere, has a standard renewal type proof which is implicit in the proofs of Lemma 2.1 and the $c>1$ part of Theorem 2.1 of [1]

LEMma 2.3. Let $Y=Y_{1}, Y_{2}, \ldots$ be independent and identically distributed nonnegative random variables such that $E Y_{1}^{p}=\infty$ for some $p \in(0,1)$. Put $S_{n}=\sum_{i=1}^{n} Y_{i}$, and, for $t \geqslant 0$, let $N_{t}(Y)=\inf \left\{n: S_{n}>t\right\}$. Then

$$
\limsup _{k \rightarrow \infty} P\left(Y_{N_{k}(Y)}>k\right)>0 \text {. }
$$

Now define random variables $W_{i}, i \geqslant 1$, by $W_{1}=T_{\lambda, r}$, and, for $i>1, W_{i}=$ $\inf \left\{t \geqslant W_{i-1}+1: X(t)-X\left(W_{i-1}\right) \notin\left(\lambda\left(t-W_{i-1}\right)^{1 / \alpha}, r\left(t-W_{i-1}\right)^{1 / \alpha}\right)\right\}$. Here $\lambda$ is arbitrary and $r=r(\lambda)$ is the $r$ of Lemma 2.2. Let $Z_{i}=W_{i}-W_{i-1}, i \geqslant 1$. Then by scaling, we get $P\left(Z_{N_{k}(Z)}>k\right) \leqslant P E(\lambda, r, k)$, and this, together with the last lemma, gives (2.5).

3. Symmetric stable processes. In this section (1.2) will be proved. The outline of the proof is the same as that of the proof just given, but new difficulties arise because we are no longer dealing with increasing processes. For a number $\alpha \in(0,2)$, let $Z_{\alpha}(t)=Z(t), t \geqslant 0$, be the symmetric stable process for which the characteristic function $f$ of $Z(1)$ is given by

$$
\log f(u)=\int_{-\infty}^{\infty}\left(e^{i u x}-1-\frac{i u x}{1+x^{2}}\right) \frac{d x}{|x|^{1+\alpha}} .
$$

Any symmetric stable process of index $\alpha$ is identical in law to a constant multiple of $Z(t)$. We will prove the following theorem.

THEOREM 3.1. Given $\mu>0$ there is an $s>0$ such that

$$
P\left(\exists t \in[0,1]: \mu h^{1 / \alpha} \leqslant Z(t+h)-Z(t) \leqslant s h^{1 / \alpha} \forall h \in(0,1]\right)>0 .
$$


Once the analog of Lemma 2.2 has been established, the proof of Theorem 3.1 follows in exactly the same manner that the proof of Theorem 2.1 followed from Lemma 2.2. The next few lemmas are part of this analog.

Given $\delta>0$, we decompose $Z(t)=U^{\delta}(t)+M^{\delta}(t)+L^{\delta}(t)$ where $U^{\delta}, M^{\delta}$ and $L^{\delta}$ are independent processes, each with stationary independent increments and for which the logarithms of the characteristic functions of $U^{\delta}(1), M^{\delta}(1)$ and $L^{\delta}(1)$ are

$$
\int_{\delta}^{\infty}\left(e^{i u x}-1\right) d x / x^{\alpha+1}, \quad \int_{-\delta}^{\delta}\left(e^{i u x}-1-\frac{i u x}{1+x^{2}}\right) d x /|x|^{\alpha+1},
$$

and

$$
\int_{-\infty}^{-\delta}\left(e^{i u x}-1\right) d x /|x|^{\alpha+1}
$$

respectively. Thus $U^{\delta}, M^{\delta}$ and $L^{\delta}$ are the processes composed of the jumps of $Z$ of sizes exceeding $\delta$, in $[-\delta, \delta]$, and less than $-\delta$, respectively. Again, we consider $\alpha$ to be fixed.

LemMa 3.2. Given $\varepsilon, \delta$ positive,

(i) $P\left(U^{\delta}(1)=0\right)=P\left(L^{\delta}(1)=0\right)=c(\delta)>0$, and

(ii) $P\left(\sup _{0 \leqslant t \leqslant 1}\left|M^{\delta}(t)\right|<\varepsilon\right)=k(\delta, \varepsilon)>0$.

Proof. Clearly $c(\delta)=\exp \left(-\int_{\delta}^{\infty} t^{-(1+\alpha)} d t\right)$. To prove (ii), note that if $k(\delta, \varepsilon)=0$ for some $\delta, \varepsilon$ positive then $k(y, \varepsilon)$ would be 0 for all $y>0$, since if $y<\delta$, there is a positive probability that the process $M^{\delta}(t)-M^{y}(t), 0 \leqslant t \leqslant 1$, is identically 0 , and this process is independent of $M^{y}(t), 0 \leqslant t \leqslant 1$. But $M^{y}(t), 0 \leqslant t \leqslant 1$, is a martingale, and thus using a standard inequality of Doob [2, p. 317], applied to continuous parameter martingales, we get

$$
\begin{aligned}
P\left(\sup _{0 \leqslant t \leqslant 1}\left|M^{y}(t)\right| \geqslant \varepsilon\right) & \leqslant \varepsilon^{-2} E\left(\sup _{0 \leqslant t \leqslant 1} M^{y}(t)\right)^{2} \leqslant 4 \varepsilon^{-2} E M^{y}(1)^{2} \\
& =4 \varepsilon^{-2} \int_{-y}^{y} x^{2}|x|^{-(1+\alpha)} d x \rightarrow 0 \quad \text { as } y \rightarrow 0,
\end{aligned}
$$

so that $k(y, \varepsilon)$ is not zero for all $y>0$, establishing (ii).

Lemma 3.3. Let $\delta \leqslant u<t$. Then

$$
P\left(u<U^{\delta}(1)<t\right) \geqslant c(\delta) \int_{u}^{t} x^{-(1+\alpha)} d x,
$$

where $c(\delta)$ is as in Lemma 3.2 .

Proof. The probability that $U^{\delta}(t), 0 \leqslant t \leqslant 1$, makes exactly one jump of size between $x$ and $x+d x$, if $x>\delta$, and no other jumps, is $c(\delta) x^{-(1+\alpha)} d x$. Thus $c(\delta) \int_{u}^{t} x^{-(1+\alpha)} d x$ is the probability that $U^{\delta}(1)$ equals the only jump made by $U^{\delta}(t)$, $0 \leqslant t \leqslant 1$, and that this jump has size between $u$ and $t$. 
Now for $x \geqslant 0$ let $P_{(x, y)}$ and $E_{(x, y)}$ denote probability and expectation associated with $Z(t)$ given $Z(x)=y$, and let $P$ and $E$ continue to stand for $P_{(0,0)}$ and $E_{(0,0)}$. Let $\mu \geqslant 1$ be considered fixed, and put $v=v(x)=(x / 2 \mu)^{\alpha}$ and $a=a(x)=v(x)-1$.

Lemma 3.4. There is a positive constant $\eta=\eta(\mu)$ such that if $x \geqslant 3 \mu$ and $y \geqslant 1$ then

$$
\begin{aligned}
P_{(1 . x)}(Z(v)- & x \in\left(x y-2^{-1} x, 2 x y+2^{-1} x\right), \\
& \left.\sup _{1 \leqslant t \leqslant v} Z(t)<Z(v)+2^{-1} x, \inf _{1 \leqslant t \leqslant v} Z(t)-x>x / 2\right) \geqslant \eta y^{-\alpha} .
\end{aligned}
$$

Proof. The process $Z(t+1)-x, t \geqslant 0$, has the same distribution under $P_{(1, x)}$ that $Z(t), t \geqslant 0$, has under $P$. Using this and the scaling property of $Z(t)$ we get that the probability in (3.1) is equal to

$$
\begin{aligned}
& P\left(Z(1) \in\left(a^{-1 / \alpha}\left(x y-2^{-1} x\right), a^{-1 / \alpha}\left(2 x y+2^{-1} x\right)\right),\right. \\
& \left.\quad \sup _{0 \leqslant t \leqslant 1} Z(t)<Z(1)+a^{-1 / \alpha} x / 2, \inf _{0 \leqslant x \leqslant 1} Z(t)>-a^{-1 / \alpha} x / 2\right) .
\end{aligned}
$$

Now, since $x \geqslant 3 \mu$, we have $l(x) \leqslant a \leqslant v(x)$, where $l(x)=x^{\alpha} \mu^{-\alpha}\left(2^{-\alpha}-3^{-\alpha}\right)$. For any $\theta>0$, recalling that $M^{\theta}, U^{\theta}$ and $L^{\theta}$ are independent, we have that the probability (3.2) is at least

$$
P\left(U^{\theta}(1) \in\left(a^{-1 / \alpha} x y, 2 a^{-1 / \alpha} x y\right)\right) P\left(L^{\theta}(1)=0\right) P\left(\sup _{0 \leqslant l \leqslant 1}\left|M^{\theta}(t)\right|<a^{-1 / \alpha} x / 4\right) .
$$

The third of the probabilities in (3.3) is at least $k\left(\theta, v(x)^{-1 / \alpha} x / 4\right)=k(\theta, \mu / 2)$, the second is $c(\theta)$, and if $v(x)^{-1 / \alpha} x y \geqslant \theta$, Lemma 3.3 gives that the first is

$$
\inf _{b \in[l(x), v(x)]} c(0) \int_{h^{-1 / \alpha} x y}^{2 b^{-1 / \alpha} x y} t^{-(1+\alpha)} d t
$$

This infimum equals $c(\theta) \Delta y^{-\alpha}$, where $\Delta$ is a positive constant depending only on $\mu$. Thus, by taking $\theta=v(x)^{-1 / \alpha} x=2 \mu$, guaranteeing $v(x)^{-1 / \alpha} x y \geqslant \theta$ since $y \geqslant 1$, we have that $\eta$ may be taken to be $k(2 \mu, \mu / 2) c(2 \mu)^{2} \Delta$.

Lemma 3.5. Given $K>0$ there exists $e=e(\mu, K)<\infty$ and $p=p(\mu, K) \in(0,1)$ such that, for $x \geqslant 3 \mu$,

$$
E_{(1, x)} Z(v)^{\alpha p} I\left(\mu v^{1 / \alpha}<Z(t)<e v^{1 / \alpha} \text { for all } t \in[1, v], Z(v)>3 \mu v^{1 / \alpha}\right) \geqslant K x^{\alpha p} .
$$

Proof. We first observe that, if $Z(1)=x$ and $\inf _{1 \leqslant t \leqslant v} Z(t)-x>-x / 2$, then $Z(t)>\mu v^{1 / \alpha}=x / 2$ for $1 \leqslant t \leqslant v$. Let

$$
H=Z(v) I\left(\sup _{1 \leqslant t \leqslant v} Z(t)<Z(v)+x / 2, \inf _{1 \leqslant i \leqslant v} Z(t)-x>-x / 2\right) .
$$


Then (3.1) implies that

$$
P_{(1, x)}(x y<H<4 x y) \geqslant \eta y^{-\alpha} \text { for each } y \geqslant 1,
$$

giving

$$
P_{(1, x)}\left(4^{k} x<H<4^{k+1} x\right) \geqslant \eta 4^{-\alpha k} \text { for } k \geqslant 0 .
$$

Let $m$ be the smallest integer such that $4^{m} x>3 \mu v^{1 / \alpha}$. Then

$$
E_{(1, x)} H^{\alpha p} I\left(4^{m} x<H<4^{n} x\right) \geqslant \sum_{k=m}^{n-1}\left(4^{k} x\right)^{\alpha p} 4^{-\alpha k} \eta=\eta x^{\alpha p} \sum_{k=m}^{n-1}\left(4^{\alpha(p-1)}\right)^{k} .
$$

First pick $p$ to be that number in $(0,1)$ such that $\eta \sum_{k=m}^{\infty}\left(4^{\alpha(p-1)}\right)^{k}=2 K$, and then let $N=N(\mu, K)$ be the smallest integer such that $\eta \sum_{k=m}^{N-1}\left(4^{\alpha(p-1)}\right)^{k}>K$. Define $e$ by $e v^{1 / \alpha}=4^{N} x+(x / 2)$. Then (3.4) holds, since for this choice of $e$, the expectation in (3.4) is at least as large as $E_{(1, x)} H^{\alpha p} I\left(4^{m} x<H<4^{N} x\right)$; in fact there is a pointwise inequality between the random variables involved in these expectations.

We need a version of (3.4) for $Z$ given $Z(s)=x$. It is the following: Let $s>0$ and $x \geqslant 3 \mu s^{1 / \alpha}$. Then for $K>0$ and $p=p(\mu, K)$ and $e=e(\mu, K)$ as in the last lemma,

$$
\begin{array}{r}
E_{(s, x)} Z(v)^{p \alpha} I\left(\sup _{s \leqslant t \leqslant v} Z(t)<e v^{1 / \alpha}, \inf _{s \leqslant t \leqslant v} Z(t)>\mu v^{1 / \alpha},\right. \\
\left.Z(v)>3 \mu v^{1 / \alpha}\right) \geqslant K x^{\alpha p} .
\end{array}
$$

Inequality (3.5) can be proved just as (3.4) was proved, or can be derived from (3.4) by using the fact that, under $P_{(a, b)}$, the distribution of $Z(t+a), t \geqslant 0$, is the same as the distribution of $b+Z(t), t \geqslant 0$, under $P$, and changing scale.

Given $K>0$ define times $\gamma_{i}(\mu, K)=\gamma_{i}, i \geqslant 0$, by $\gamma_{0}=1, \gamma_{1}=v(Z(1))=$ $(Z(1) / 2 \mu)^{\alpha}$ if $3 \mu<Z(1)<e, \gamma_{1}=\gamma_{0}$ otherwise, and, for $k \geqslant 1, \gamma_{k+1}=v\left(Z\left(\gamma_{k}\right)\right)$ on

$$
\begin{aligned}
& \left\{\gamma_{k}>\gamma_{k-1}, \mu v\left(\gamma_{k}\right)^{1 / \alpha}<Z(t)<e v\left(\gamma_{k}\right)^{1 / \alpha} \text { for all } t \in\left[\gamma_{k-1}, \gamma_{k}\right],\right. \\
& \left.\qquad Z\left(\gamma_{k}\right) \in\left(3 \mu \gamma_{k}^{1 / \alpha}, e \gamma_{k}^{1 / \alpha}\right)\right\}
\end{aligned}
$$

and $\gamma_{k+1}=\gamma_{k}$ otherwise. Here $e=e(K)$ is as in the last lemma. Let $\gamma=\lim _{k \rightarrow \infty} \gamma_{k}$ and $N=\sup \left\{k: \gamma_{k}>\gamma_{k-1}\right\}$. Then

$$
\gamma_{N-1} \leqslant V(\mu, K)=V=\inf \left\{t \geqslant 1: Z(t) \notin\left(\mu t^{1 / \alpha},\left(e^{2} / 2 \mu\right) t^{1 / \alpha}\right)\right\},
$$

and furthermore $\gamma_{N-1} \geqslant(2 \mu / e)^{\alpha} \gamma_{N}$. We will show that, for large enough $K, E \gamma^{p}=$ $\infty$, where $p=p(\mu, K)$ is as above, which implies $E V^{p}=\infty$. From this point the proof of Theorem 3.1 can be completed in exactly the same way the proof of Theorem 2.1 was finished off after Lemma 2.2 had been proved. 
Let $\beta=\left(1-(2 / 3)^{\alpha}\right)$. Then $\left(\gamma_{k+1}-\gamma_{k}\right) \geqslant \beta \gamma_{k+1}$ on $\left\{\gamma_{k+1}>\gamma_{k}\right\}$, so that

$$
\begin{aligned}
& E\left(\gamma_{k+1}-\gamma_{k}\right)^{p} \geqslant \beta E \gamma_{k+1}^{p} I\left(\gamma_{k+1}>\gamma_{k}\right)=\beta E\left(Z\left(\gamma_{k}\right) / 2 \mu\right)^{\alpha p} I\left(\gamma_{k+1}>\gamma_{k}\right) \\
& =\beta E\left(Z\left(\gamma_{k}\right) / 2 \mu\right)^{\alpha p} I\left(\gamma_{k}>\gamma_{k-1}, \mu \gamma_{k}^{1 / \alpha}<Z(t)<e \gamma_{k}^{1 / \alpha}\right. \\
& \text { for } \left.\gamma_{k-1} \leqslant t \leqslant \gamma_{k}, Z\left(\gamma_{k}\right) \in\left(3 \mu \gamma_{k}^{1 / \alpha}, e \gamma_{k}^{1 / \alpha}\right)\right) \\
& =\beta E E\left[( Z ( \gamma _ { k } ) / 2 \mu ) ^ { \alpha p } I \left(\gamma_{k}>\gamma_{k-1}, \mu \gamma_{k}^{1 / \alpha}<Z(t)<e \gamma_{k}^{1 / \alpha}\right.\right. \\
& \text { for } \gamma_{k-1} \leqslant t \leqslant \gamma_{k} \text {, } \\
& \text { and } \left.\left.Z\left(\gamma_{k}\right) \in\left(3 \mu \gamma_{k}^{1 / \alpha}, e \gamma_{k}^{1 / \alpha}\right)\right) \mid \mathscr{F}_{\gamma_{k-1}}^{Z}\right]
\end{aligned}
$$

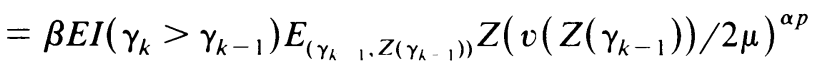

$$
\begin{aligned}
& \cdot I\left(\mu v\left(Z_{\gamma_{k-1}}\right)<Z(t)<e v\left(Z_{\gamma_{k-1}}\right) \text { for } \gamma_{k-1} \leqslant t \leqslant v\left(Z\left(\gamma_{k-1}\right)\right)\right. \text {, } \\
& \left.Z\left(v\left(\gamma_{k-1}\right)\right) \in\left(3 \mu v\left(Z\left(\gamma_{k-1}\right)\right)^{1 / \alpha}, e v\left(Z\left(\gamma_{k-1}\right)\right)^{1 / \alpha}\right)\right) \\
& \geqslant \beta E I\left(\gamma_{k}>\gamma_{k-1}\right) K Z\left(\gamma_{k-1}\right)^{\alpha p}(2 \mu)^{-\alpha p} \quad \text { (using (3.5)) } \\
& \geqslant \beta E I\left(\gamma_{k}>\gamma_{k-1}\right) K\left(2 \mu \gamma_{k}^{1 / \alpha}\right)^{\alpha p}(2 \mu)^{-\alpha p} \\
& =K \beta E \gamma_{k}^{p} I\left(\gamma_{k}>\gamma_{k-1}\right) \geqslant K \beta E\left(\gamma_{k}-\gamma_{k-1}\right)^{p} \text {. }
\end{aligned}
$$

Thus, if $K>2 \beta^{-1}$, then $E\left(\gamma_{k+1}-\gamma_{k}\right)^{p}>2 E\left(\gamma_{k}-\gamma_{k-1}\right)^{p}$ so that $E\left(\gamma_{k+1}-\gamma_{k}\right)^{p} \rightarrow$ $\infty$ and thus $E \gamma^{p}=\infty$.

4. Hausdorff dimension. Let $X(t), t \geqslant 0$, be a strictly stable subordinator of index $\alpha$, as in $\S 2$. Put $A=\left\{t: \liminf _{h \downarrow}(X(t+h)-X(t)) h^{-1 / \alpha}=+\infty\right\}$. In this section we prove

TheOREM 4.1. The Hausdorff dimension of $A$ is almost surely zero.

The reader has now seen that none of the times $t$ which were found to show the truth of Theorem 2.1 are necessarily in $A$. The analog of Theorem 4.1 for symmetric stable processes is true. For exponents $\alpha \in(0,1)$ this follows immediately from Theorem 4.1. We do not give the proof of this analog, since it closely parallels the proof of Theorem 4.1.

Proof of Theorem 4.1. For $c>0$ let $A_{c}=\left\{t: \liminf _{h \downarrow}(X(t+h)-X(t)) h^{-1 / \alpha}\right.$ $>c\}$, and let $\tau_{c}=\inf \left\{t \geqslant 1: X(t) \leqslant c(t-1)^{1 / \alpha}\right\}$. Let the Hausdorff dimension of a set $B$ be denoted by $\operatorname{dim} B$. Theorem 4.1 will be proved by showing that, given $\delta>0$, there is a $c$ such that $\operatorname{dim} A_{c}<\delta$. To do this we employ

Lemma 4.2. Given $p \in(0,1)$ there exists $c \in(0, \infty)$ such that $E \tau_{c}^{p}<\infty$.

Before proving Lemma 4.2, we will show how it implies Theorem 4.1. Let, for each positive integer $j, A_{c}^{j}=\left\{t \geqslant 0: X(t+h)-X(t)>c h^{1 / \alpha}\right.$ for $\left.0<h \leqslant j^{-1}\right\}$. We will show that $E \tau_{c}^{p}<\infty$ implies that $\operatorname{dim} A_{c}^{j} \leqslant 1-p$ a.s., which immediately gives $\operatorname{dim} A_{c} \leqslant 1-p$ a.s. Since the proof of this is the same for all positive integers $j$, we prove it for $j=1$. To do this it suffices to show that $\operatorname{dim}\left(A_{c}^{1} \cap[0,1)\right) \leqslant 1-p$ a.s. 
We note that if $I=\left[a, a+n^{-1}\right)$ is a subinterval of $[0,1)$ of length $n^{-1}$, then

$$
\left\{A_{c}^{1} \cap I \neq \varnothing\right\} \subset\left\{X_{t+a}-X_{t}>c\left(t-\left(a+n^{-1}\right)\right)^{1 / \alpha},\left(a+n^{-1}\right) \leqslant t \leqslant a+n^{-1}+1\right\}
$$

and changing scale gives

$$
P\left(A_{c}^{1} \cap I \neq \varnothing\right\} \leqslant P\left(\tau_{c}>n\right) \leqslant n^{-p} E \tau_{c}^{p}
$$

Thus, if $N$ is the number of intervals of the form $\left[j n^{-1},(j+1) n^{-1}\right), 0 \leqslant j \leqslant n-1$, which have nonempty intersection with $A_{c}^{1}$, we have $E N \leqslant n n^{-p} E \tau_{c}^{p}$. Using the above intervals as a covering of $A_{c}^{1}$, we have $A_{c}^{1}$ covered by $N$ intervals of length $n^{-1}$. The expected sum of the lengths of these intervals raised to the $\delta$ power is $n^{-\delta} E N$, so the expected sum goes to 0 as $n$ goes to $\infty$ whenever $\delta>1-p$, which easily gives $\operatorname{dim}\left(A_{c}^{1} \cap[0,1)\right) \leqslant 1-p$ a.s.

Now we turn to the proof of Lemma 4.2. Let $T_{c}=\inf \left\{t \geqslant 1: X(t) \leqslant c t^{1 / \alpha}\right\}$. We will show that, given $p \in(0,1)$, there is a $k=k(p)$ such that $E T_{k}^{p}<\infty$. Since $\tau_{k^{\prime}}$ does not exceed $\tau_{k}$ plus a constant if $k^{\prime}>k$, this will establish Lemma 4.2.

First we note that $E X(1)^{\alpha p}<\infty$ if $p<1$. One way to show this is to divide $X(1)=B+L$, where $B$ is the sum of the jumps of $X(t), 0 \leqslant t \leqslant 1$, of magnitude exceeding one and $L$ is the sum of the jumps of magnitude in $(0,1)$. Then $E L<\infty$, and $E B^{\alpha p} \leqslant E \sum j^{\alpha p}<\infty$, where the sum is taken over all the jumps $j$ making up $B$. Now given $x$ and $c$ positive, we have

$$
\begin{array}{rl}
E_{(1, x)} X\left(x^{\alpha}\right)^{\alpha p} & I\left(X\left(x^{\alpha}\right)>c x\right)=E\left(X\left(x^{\alpha}-1\right)+x\right)^{\alpha p} I\left(X\left(x^{\alpha}-1\right)+x>c x\right) \\
& \leqslant E\left(X\left(x^{\alpha}\right)+x\right)^{\alpha p} I\left(X\left(x^{\alpha}\right)>(c-1) x\right) \\
& \leqslant 2^{\alpha p} E X\left(x^{\alpha}\right)^{\alpha p} I\left(X\left(x^{\alpha}\right)>(c-1) x\right)+2^{\alpha p} x^{\alpha p} I\left(X\left(x^{\alpha}\right)>(c-1) x\right) \\
& \leqslant 2^{\alpha p} x^{\alpha p} E X(1)^{\alpha p} I(X(1)>c-1)+2^{\alpha p} x^{\alpha p} P(X(1)>c-1) .
\end{array}
$$

Pick $k=k(p)$ so large that this last quantity does not exceed $x^{\alpha p} / 3$ for $c=k$, and assume $k \geqslant 2^{\alpha}$.

Now let $\eta_{0}=1$, and $\eta_{1}=X(1)^{\alpha}$ if $X(1)>k, \eta_{1}=1$ otherwise, and, in general, $\eta_{i+1}=X\left(\eta_{i}\right)^{\alpha}$ if $\eta_{i}>\eta_{i+1}$ and $X\left(\eta_{i}\right)>k \eta_{i}^{1 / \alpha}, \eta_{i+1}=\eta_{i}$ otherwise. Let $\eta=\lim \eta_{i}$, and note that $\eta \geqslant T_{k}$. Now

$$
\begin{aligned}
E\left(\eta_{i+1}-\eta_{i}\right)^{p} & \leqslant E \eta_{i+1}^{p} I\left(X\left(\eta_{i}\right)>k \eta_{i}^{1 / \alpha}, \eta_{i+1}>\eta_{i}\right) \\
& =E X\left(\eta_{i}\right)^{\alpha p} I\left(X\left(\eta_{i}\right)>k \eta_{i}^{1 / \alpha}, \eta_{i+1}>\eta_{i}\right) \\
& =E E\left(X\left(\eta_{i}\right)^{\alpha p} I\left(X\left(\eta_{i}\right)>k \eta_{i}^{1 / \alpha}, \eta_{i+1}>\eta_{i}\right) \mid \mathscr{F}_{\eta_{i-1}}\right) \\
& =E I\left(\eta_{i}>\eta_{i-1}\right) E_{\left(\eta_{i-1}, X\left(\eta_{i-1}\right)\right.}, X\left(\eta_{i}\right)^{\alpha p} I\left(X\left(\eta_{i}\right)>k \eta_{i}^{1 / \alpha}\right) \\
& \leqslant E I\left(\eta_{i}>\eta_{i-1}\right) X\left(\eta_{i-1}\right)^{\alpha p} / 3 \\
& \leqslant E I\left(\eta_{i}>\eta_{i-1}\right) \eta_{i}^{p} / 3 \leqslant 2 E\left(\eta_{i}-\eta_{i-1}\right)^{p} / 3
\end{aligned}
$$


the last inequality holding since $\eta_{i}-\eta_{i-1}>\eta_{i} / 2$ on $\left\{\eta_{i}>\eta_{i-1}\right\}$, since $k>2^{\alpha}$. Thus

$$
\begin{aligned}
E_{\eta}^{p} & =E\left(1+\sum_{i=1}^{\infty}\left(\eta_{i}-\eta_{i-1}\right)^{p}\right) \leqslant 1+\sum_{i=1}^{\infty} E\left(\eta_{i}-\eta_{i-1}\right)^{p} \\
& \leqslant 1+\sum_{j=1}^{\infty}(2 / 3)^{i} E\left(\eta_{1}-1\right)^{p}<\infty .
\end{aligned}
$$

Acknowledgement. The author thanks Ed Perkins for pointing out the problems in this area, and Herman Rubin, for a number of discussions about stable processes.

\section{REFERENCES}

I. B. J. Davis, On Brownian slow points, Z. Wahrsch. Verw. Gebiete (to appear).

2. J. J. Doob, Stochastic processes, Wiley, New York, 1953.

3. A. Dvoretsky, On the oscillation of the Brownian motion process, Israel J. Math. 1 (1963), 212-214.

4. Bert Fristedt, Uniform local behavior of stable subordinators, Ann. Probab. 7 (1979), 1003-1013.

5. P. Greenwood and E. Perkins, A conditioned limit theorem for random walk, and Brownian local time on square root boundaries, Ann. Probab. 11 (1983), 227-261.

6. J. P. Kahane, Sur l' irrégularité locale du mouvement brownien, C. R. Acad. Sci. Paris. Sér. 278 (1974), 331-333.

7. E. Perkins, On the Hausdorff measure of the Brownian slow points, Z. Wahrsch. Verw. Gebiete (to appear).

Department of Statistics, Purdue University, West Lafayette, Indiana 47907 